\title{
BMJ Open Evaluating the impacts of screening and smoking cessation programmes on lung cancer in a high-burden region of the USA: a simulation modelling study
}

\author{
Angela C Tramontano, ${ }^{1}$ Deirdre F Sheehan, ${ }^{1}$ Pamela M McMahon, ${ }^{1,2}$ \\ Emily C Dowling, ${ }^{1}$ Theodore R Holford, ${ }^{3}$ Karen Ryczak, ${ }^{4}$ Samuel M Lesko, ${ }^{4}$ \\ David T Levy, ${ }^{5}$ Chung Yin Kong ${ }^{1,2}$
}

To cite: Tramontano AC, Sheehan DF, McMahon PM, et al. Evaluating the impacts of screening and smoking cessation programmes on lung cancer in a high-burden region of the USA: a simulation modelling study. BMJ Open 2016;6:e010227. doi:10.1136/bmjopen-2015010227

- Prepublication history for this paper is available online. To view these files please visit the journal online (http://dx.doi.org/10.1136/ bmjopen-2015-010227).

ACT and DFS contributed equally.

Received 9 October 2015 Revised 2 February 2016 Accepted 9 February 2016

CrossMark

For numbered affiliations see end of article.

Correspondence to Dr Chung Yin Kong; joey@mgh-ita.org

\section{ABSTRACT}

Objective: While the US Preventive Services Task Force has issued recommendations for lung cancer screening, its effectiveness at reducing lung cancer burden may vary at local levels due to regional variations in smoking behaviour. Our objective was to use an existing model to determine the impacts of lung cancer screening alone or in addition to increased smoking cessation in a US region with a relatively high smoking prevalence and lung cancer incidence.

Setting: Computer-based simulation model.

Participants: Simulated population of individuals 55 and older based on smoking prevalence and census data from Northeast Pennsylvania.

Interventions: Hypothetical lung cancer control from 2014 to 2050 through (1) screening with CT, (2) intensified smoking cessation or (3) a combination strategy.

\section{Primary and secondary outcome measures:}

Primary outcomes were lung cancer mortality rates. Secondary outcomes included number of people eligible for screening and number of radiation-induced lung cancers.

Results: Combining lung cancer screening with increased smoking cessation would yield an estimated $8.1 \%$ reduction in cumulative lung cancer mortality by 2050. Our model estimated that the number of screening-eligible individuals would progressively decrease over time, indicating declining benefit of a screening-only programme. Lung cancer screening achieved a greater mortality reduction in earlier years, but was later surpassed by smoking cessation.

Conclusions: Combining smoking cessation programmes with lung cancer screening would provide the most benefit to a population, especially considering the growing proportion of patients ineligible for screening based on current recommendations.

\section{INTRODUCTION}

Lung cancer is the leading cause of cancerrelated death in the USA, resulting in

\section{Strengths and limitations of this study}

- To our knowledge, this is the first study to model the impact of combining lung cancer screening and smoking cessation programmes in the USA.

- Our study demonstrates the use of simulation modelling as a critical resource to help translate national guidelines into local programmes, optimising cancer control efforts for a specific population.

- We assumed that current screening and treatment guidelines will remain the same; therefore, actual reduction rates may be different if future policies change. However, our results on the benefits of a long-term cessation programme are promising.

approximately 150000 deaths per year. ${ }^{1}$ The National Lung Screening Trial (NLST), a randomised trial in the USA involving more than 53000 current and former heavy smokers aged 55-74, found 20\% fewer lung cancer deaths among trial participants screened with three annual low-dose CT versus three annual chest radiography examinations. ${ }^{2}{ }^{3}$ Based on the NLST results, the US Preventive Services Task Force (USPSTF) recommended annual CT screening for eversmokers aged 55-80 with at least 30 packyears of smoking history and no more than 15 years since quitting. ${ }^{4}$ In early 2015 , the Centers for Medicare and Medicaid Services (CMS) also released a decision memo in support of low-dose CT screening coverage for eligible individuals. ${ }^{5}$ As a result, nationwide adoption of lung cancer screening is currently being implemented in the USA.

Although CT screening has been recommended by the USPSTF and CMS, the potential mortality reduction due to lung cancer 
screening as a stand-alone programme is limited. The $20 \%$ lung cancer mortality reduction seen in the NLST was estimated among people who were screened, and the lung cancer mortality reduction of screening with CT among the whole US population has yet to be established. The disease burden prevented by lung cancer screening with CT may also differ by geographical location because there exists much regional variation in smoking patterns and lung cancer rates. ${ }^{167}$ For example, smoking prevalence is significantly higher in Northeast (NE) Pennsylvania compared with the USA as a whole, a finding typical of areas with low income and education. ${ }^{8-10}$ In NE Pennsylvania, the incidence of lung and bronchus cancer is also higher in men and is increasing in women compared with rates among the US population. ${ }^{11}$

In this study, we utilised the Massachusetts General Hospital's Lung Cancer Policy Model (LCPM) to determine the optimal lung cancer control policy in NE Pennsylvania, where $29 \%$ of adults are current smokers. ${ }^{12}$ We estimated the impacts of implementing lung cancer screening alone or incorporating an additional smoking cessation intervention on lung cancer mortality. Our results can aid policymakers' development of lung cancer control policy in NE Pennsylvania and in other areas with high prevalence of current and former smokers.

\section{MATERIALS AND METHODS Model overview}

The LCPM is a state-transition microsimulation model that simulates an individual patient's lung cancer development, progression, detection, follow-up, treatment, and survival. ${ }^{13} 14$ The LCPM has been used previously to evaluate the impact of tobacco control strategies on mortality reduction, ${ }^{15}$ as well as the cost-effectiveness of imaging-based screening programmes for lung cancer. ${ }^{17}$ Owing to concerns that chest CT examinations will detect incidental pulmonary nodules and result in invasive testing for benign or indolent disease, the LCPM explicitly models benign nodules and detailed clinical events. Each hypothetical individual in the LCPM can develop up to three cancers from any of the following five lung cancer cell types: adenocarcinoma (including adenocarcinoma in situ), large cell, squamous cell, small cell and other. The lung cancer risks, incidence rates and disease progression rates vary by histological type. Lung cancer risk and development in each simulated individual depends on a complete smoking history that includes length of time a person smoked and cigarettes smoked per day; both current and former smokers are at risk of developing lung cancer based on smoking history. The model outputs of the LCPM have been calibrated and validated using results from the NLST and the Prostate, Lung, Colorectal and Ovarian Cancer Screening Trial (PLCO). ${ }^{18}$

The LCPM also incorporates the radiation risk equations specified in the Biological Effects of Ionizing
Radiation (BEIR) VII report to account for the radiation exposure of screening. ${ }^{16} 171920$ The BEIR VII report derived a set of empirical radiation risk equations for multiple cancer sites primarily by fitting mathematical functions to data from Japanese atomic bomb survivors. This empirically derived model is also endorsed by the International Commission on Radiological Protection, and currently represents the most widely accepted approach to estimate cancer risks.

For additional details, the LCPM is described in an online technical appendix (http://www.cisnet.cancer. gov/lung/profiles.html).

\section{NE Pennsylvania inputs}

We developed a NE Pennsylvania version of the LCPM using data from regional surveys. The population of $\mathrm{NE}$ Pennsylvania is older in average age and has lower average household income and educational attainment than the general US population. Between 2005 and 2007, in Lackawanna and Luzerne counties, the two largest counties in the region, $18.1 \%$ and $18.3 \%$ of residents were over 65 years of age (compared with $12.5 \%$ in the USA) with median household incomes of \$41 594 and $\$ 44401$, respectively (compared with $\$ 50007$ in the USA).

Regional smoking behaviour was derived from three separate sources. First, data on smoking prevalenceincluding tobacco use, the average number of cigarettes smoked per day, and cancer screening practices-was collected by the Northeast Regional Cancer Institute (NRCI) through a population-based study of health behaviours from 2002 to 2006, using a sampling protocol similar to that used by the Behavioral Risk Factor Surveillance System (BRFSS). ${ }^{21}$ Tobacco use data in the region were also obtained from $800 \mathrm{NE}$ Pennsylvania adults in the control arm of a 2007-2011 study of colorectal cancer. $^{22}$ The third data source was the Pennsylvania BRFSS, which includes regional aggregated data for three of six counties (Lackawanna, Luzerne and Wyoming counties) within the NE Pennsylvania region. $^{12} 23$ These three counties have a combined population of 563631 , representing $78.6 \%$ of the population of the greater NE Pennsylvania region. In the Pennsylvania BRFSS, 29\% of adults in these counties aged 18 years or older were current smokers, compared with $20.4 \%$ of adults in the USA. ${ }^{21}$ Additionally, $26 \%$ of adults in the region were former smokers.

Even with three data sources for smoking behaviour, the historical smoking data for the NE Pennsylvania region did not adequately span the entire age group and time horizon of interest and was therefore supplemented with 1965-2009 data from the National Health Interview Survey (NHIS) to create smoking histories beginning with the 1910 birth cohort and projected forward until 2050, using the methods described in Holford et al. ${ }^{24}$ The probabilities of smoking initiation and cessation rates as functions of age observed in the NHIS were assumed to have similar shapes but differing 
magnitudes in NE Pennsylvania, with the effect of age on smoking assumed to be the same in the NRCI population as in NHIS. Since periods were represented by a limited time span in NE Pennsylvania, they were assumed to remain constant. The birth cohort was systematically created to pick up differences over time. The data were then smoothed using constrained cubic splines for age, period and cohort to estimate the prevalence of current smokers, former smokers and neversmokers in cohorts beginning in 1910. An interaction between NRCI and NHIS was used to estimate possible differences in cohort trend between NE Pennsylvania and the USA.

Birth cohort-specific lung cancer incidence and mortality rates in NE Pennsylvania were used to ensure that the LCPM-generated outputs were consistent with local patterns. The NRCI manages a regional cancer registry that includes information on lung cancer incidence and initial treatment from nine community hospitals. The regional registry, which is part of the Pennsylvania Cancer Registry, captures more than $95 \%$ of lung cancer cases in the NE Pennsylvania region. Incidence and mortality data are aggregated from each of the six counties to obtain age-specific rates by sex and year for the region.

\section{Lung cancer control scenarios}

The effects of different cancer control scenarios on lung cancer incidence and mortality were evaluated. In the base case scenario, there was no lung cancer screening and no change in the current smoking cessation rate in NE Pennsylvania. We compared the base case with the following three scenarios: (1) screening based on the USPSTF recommendations with $100 \%$ adherence; (2) intensified smoking cessation-through programmes such as treatment provision and intervention by healthcare providers-resulting in a $50 \%$ increase in smoking cessation rates among all smokers; and (3) screening with $100 \%$ adherence plus intensified smoking cessation.

The screening programmes were implemented in the model in 2014 and the smoking cessation programmes were assumed to result in a $50 \%$ increase in cessation rates starting in 2014. Previous analyses have demonstrated increases in quit rates near $40 \%$ for programmes that expand cessation treatment coverage and encourage clinician intervention, with increases of over $76 \%$ when cessation strategies are combined with no-cost telephone quitlines. ${ }^{25-27}$ Outcomes were projected until 2050 and results are presented for years 2020, 2030, 2040 and 2050.

We performed sensitivity analyses for screening scenarios assuming screening adherence rates of $40 \%, 60 \%$ and $80 \%$, where adherence was defined as the proportion of the eligible screening population that was adherent during a given screen. Rates were chosen based on a NHIS study finding adherence rates of $54.6 \%, 69.3 \%$, $85.8 \%$ and $46.4 \%$ in 2010 for colorectal, breast, cervical and prostate cancer screening, respectively. ${ }^{28}$
RESULTS

\section{Baseline lung cancer mortality}

The projected lung cancer mortality rates in $\mathrm{NE}$ Pennsylvania compared with the USA, with no lung cancer screening programme and no change in current smoking cessation until 2050, are displayed in figure 1 . Despite a decline in mortality rates in both $\mathrm{NE}$ Pennsylvania and the USA over the study period, the mortality rate in NE Pennsylvania remains higher than that of the USA, and the disparity between the two rates increases over time. In 2010, the lung cancer mortality rate in NE Pennsylvania was higher than the US average by 7.5 deaths per 100000 person-years, a $5.3 \%$ increase. By 2050, the difference is projected to grow to 16.9 deaths per 100000 person-years, a $44.1 \%$ increase.

\section{Eligibility for screening}

The number of individuals eligible for lung cancer screening was comparable for the screening-only and the combined screening and cessation scenarios during the first 7 years of programme adoption, as shown in figure 2. If the current cessation programme is intensified, the number of people eligible for screening may not differ from the screening-only scenario because of the ' 15 years since quitting' criterion of the USPSTF screening guidelines, but should eventually decrease accordingly. However, our model results showed that the number of eligible individuals will progressively decrease in both scenarios after 2014.

\section{Health outcomes of lung cancer control programmes}

Health outcomes of the three lung cancer control scenarios and the base case scenario in 2020, 2030, 2040 and 2050 are presented in table 1 . We estimate that in 2020 the screening-only scenario prevented 9.4 deaths per

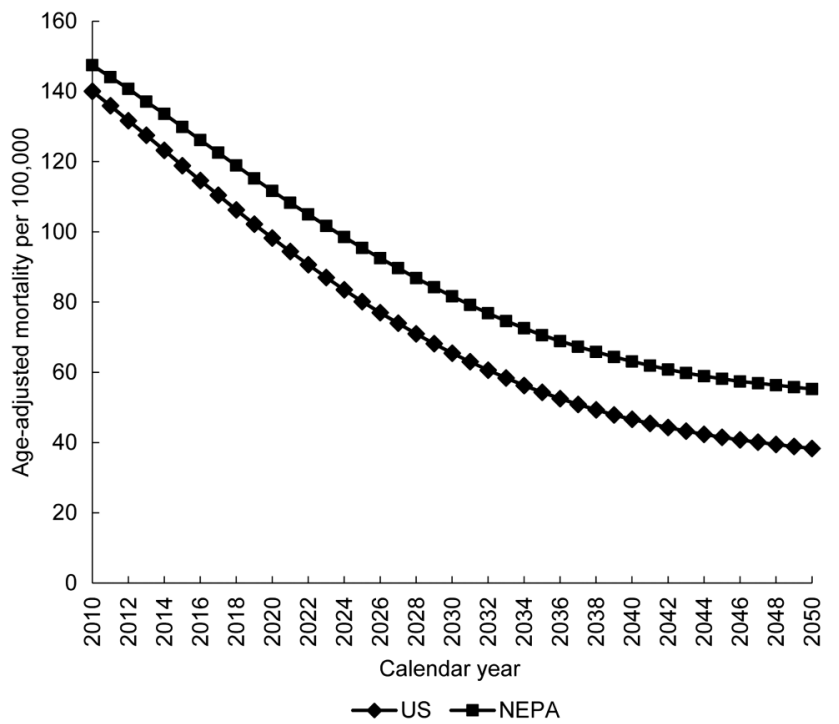

Figure 1 The projected lung cancer mortality rates for Northeast Pennsylvania (NEPA) and the USA for males and females ages 55 and older. 


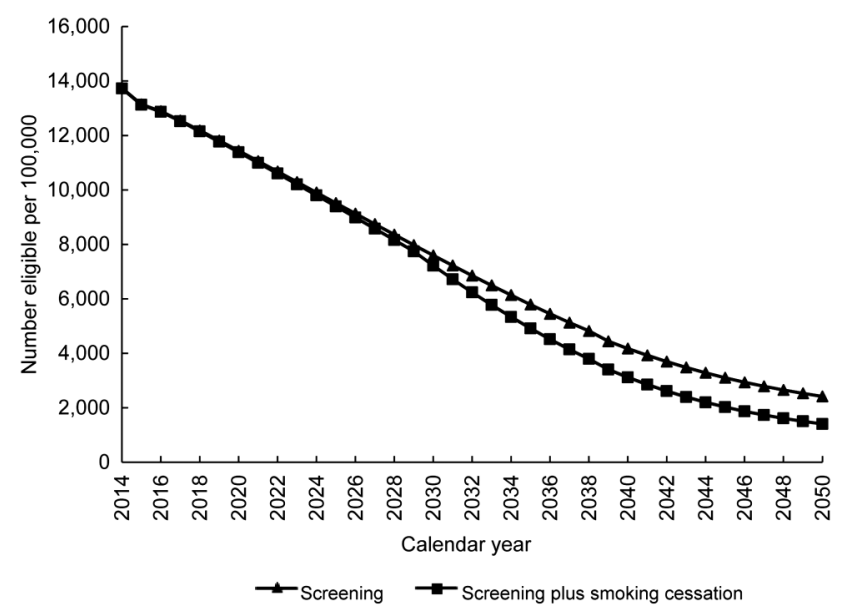

Figure 2 The number of people eligible for screening (per 100000 ) as a function of calendar year, where triangles represent a screening-only scenario and squares represent combining screening with an intensive smoking cessation programme.

100000 person-years (8.2\% reduction in lung cancer death rate), the cessation-only scenario prevented 0.3 deaths per 100000 person-years $(0.3 \%$ reduction), and the combined screening and smoking cessation scenario prevented 9.7 deaths per 100000 person-years $(8.4 \%$ reduction). In 2050, we estimate that the rates of lung cancer deaths avoided were 1.0 deaths per 100000 person-years (1.9\% reduction) for screening-only, 4.0 deaths $(7.1 \%$ reduction) for smoking cessation-only, and 4.6 deaths $(8.2 \%$ reduction) for combined screening and cessation.

To quantify overall effectiveness of the scenarios, cumulative lung cancer mortality reduction, starting in 2014, is shown in figure 3. Our results showed that the cumulative lung cancer mortality reduction of the screening-only scenario will decrease from 2020 to 2050, while the mortality reduction of the cessation-only scenario will increase.

Since ionising radiation from screening CT has the potential to induce lung cancers, it is important to quantify the potential harms from lung cancer screening programmes. In 2020, the estimated additional number of lung cancers due to radiation exposure for screened males in the screening-only strategy was 0.78 per 100000 person-years, which grew to 0.87 per 100000 personyears by 2050 .

\section{Sensitivity analysis}

We performed additional simulation runs to evaluate the impact of screening adherence on lung cancer mortality rates (table 2). In 2020, with screening adherence set at $40 \%, 60 \%$ and $80 \%$ for the screening-only scenario, the number of lung cancer deaths prevented per 100000 person-years were 3.5, 5.4 and 7.2, respectively. In 2050 , lung cancer deaths avoided for $40 \%, 60 \%$ and $80 \%$ adherence dropped to $0.2,0.5$ and 0.8 deaths per

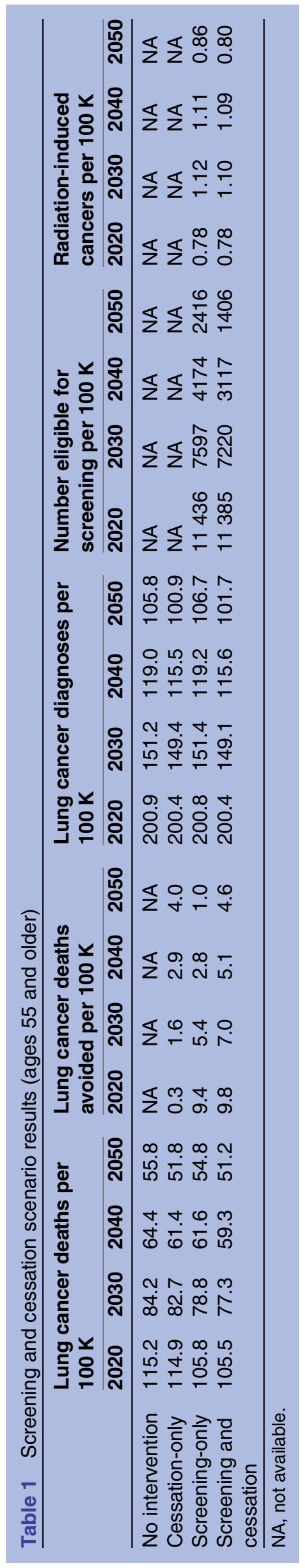




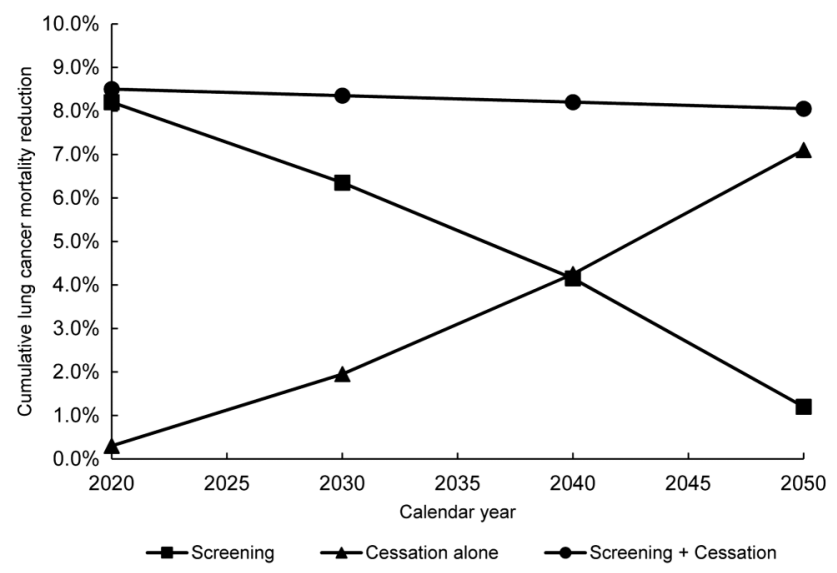

Figure 3 The cumulative lung cancer mortality reduction for screening-only, cessation-only, and combination of screening and cessation scenarios.

100000 person-years, respectively. Our results indicate that even with $60 \%$ adherence, combined screening and smoking cessation resulted in a significant reduction of lung cancer deaths.

\section{DISCUSSION}

Our study applied an existing lung cancer model to evaluate screening and cessation programmes in $\mathrm{NE}$ Pennsylvania, a region with higher smoking prevalence and lung cancer incidence than the USA overall. By using region-specific data in a model, we were able to project and estimate the lung cancer mortality reduction under multiple potential lung cancer control policies. With higher baseline smoking rates in men and women, our results from the LCPM showed that the regional lung cancer mortality from the present through the year 2050 will be higher than national trends in lung cancer mortality. Thus, it is necessary to develop a comprehensive lung cancer control policy to minimise this disparity in health outcomes. Simulation modelling can serve as a critical resource to local cancer control planners to help translate national guidelines into programmes tailored for a specific geographic area and population.

Our results showed that the number of people eligible for screening will decrease in the future, which will reduce the benefits from a CT screening-only programme. Our model prediction is consistent with a recent study which showed a decrease in the proportion of patients with lung cancer meeting USPSTF screening eligibility criteria in a Midwestern region, ${ }^{29}$ calling into question the potential long-term effectiveness of screening as a cancer control policy. A lung cancer control policy incorporating both CT screening and intensive smoking cessation may be the only option to achieve desired mortality reduction. Model predictions showed that the initial benefit of a screening-only programme compared with a cessation-only programme will eventually diminish as the number of people eligible for screening decreases. After 2040, a smoking

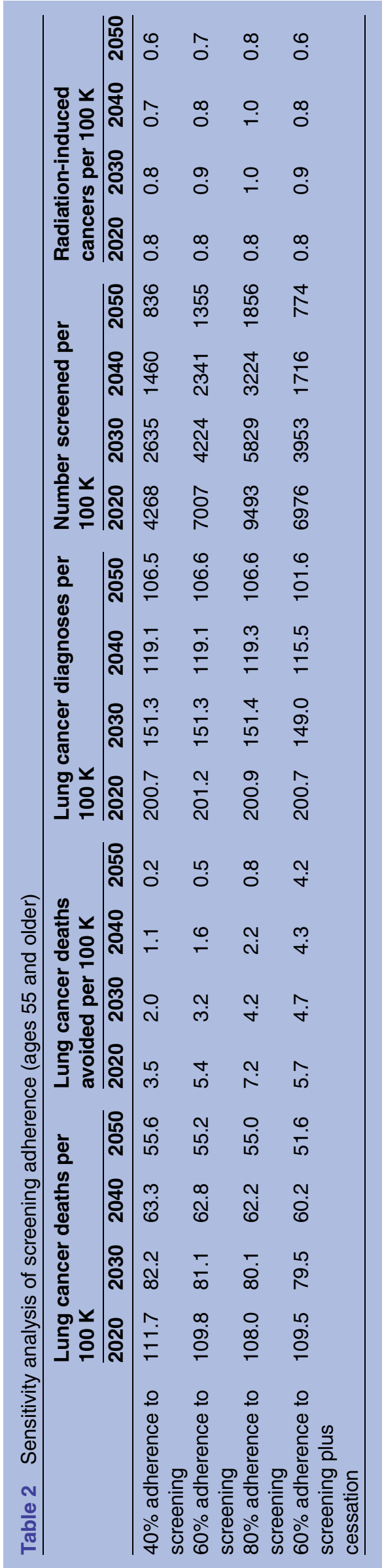


cessation-only programme will reduce the number of lung cancer deaths more than a CT screening-only programme based on current screening guidelines. Our model also predicted a radiation-induced lung cancer rate associated with CT screening of less than 2 cases per 100000 per year.

Our study reports reductions in mortality rates that are lower than the $20 \%$ observed in the NLST, because the mortality reduction in our study is based on the population of NE Pennsylvania, which includes both light smokers and non-smokers, whereas the NLST results are based on a population with restrictive eligibility criteria. The benefit of screening is diluted in the population that includes those eligible for screening as well as those at lower risk who are ineligible for screening. In addition, the NLST population was composed of healthy volunteers who knew they would be assigned to one of two screening arms. The participants were moderately younger and less likely to be current smokers, and thus were possibly healthier than the general US population. ${ }^{30}$ So, the mortality reduction observed in the NLST cannot be directly compared with our model predictions for NE Pennsylvania.

Prior studies have found an important role for programmes that increase smoking cessation, for example, through brief interventions by healthcare providers, quit lines and by providing access to treatment. ${ }^{25} 3132$ The findings of our current study are consistent with those of prior studies, and extend those of prior studies by incorporating lung cancer screening. Health policy that does not integrate cessation, but instead is based solely on CT screening of individuals who meet the eligibility criteria recommended by the USPSTF, may be increasingly inadequate due to the decreasing number of people eligible for screening under these guidelines. To our knowledge, this is the first study to model the impact of combining lung cancer screening and smoking cessation programmes in the USA.

There are several limitations to this study. First is the paucity of county-level smoking pattern data. We used multiple sources of data to estimate the regional smoking pattern, and smoking rates were smoothed using constrained cubic splines that assumed current trends would continue into the future. Recent and future implementation of tobacco control policies, however, will influence the future prevalence of smoking and incidence of lung cancer. While the effects of tobacco control policies on lung cancer rates are delayed, these policies have the potential to affect a much larger percentage of the population in the long term. Second, we chose a hypothetical programme that results in a $50 \%$ increase in cessation to demonstrate the effect that increased cessation can potentially have on lung cancer mortality in NE Pennsylvania. Previous studies have demonstrated the potential for high rates of cessation after the implementation of cessation programmes, reporting increases in quit rates near $40 \%$ for programmes that expand cessation treatment coverage and encourage clinician intervention, and increases of over $76 \%$ when cessation strategies are combined with no-cost telephone quitlines. ${ }^{25-27} 32$ We did not consider recidivism in smokers and there are currently no trials to show that cessation reduces mortality in the long term. In addition, we assumed current screening and treatment guidelines will remain the same; therefore, actual reduction rates may be different if future policies change. However, our results on the benefits of a longterm cessation programme are promising.

In conclusion, a lung cancer control strategy that incorporates CT screening among eligible ever-smokers with intensified smoking cessation will decrease lung cancer mortality at a greater rate than screening-only or cessation-only programmes. This combination strategy may be the best option for achieving lung cancer mortality reduction in NE Pennsylvania that is comparable to mortality reduction in the USA. Our study demonstrates the ability for simulation models to translate national guidelines into local programmes, optimising cancer control efforts for a specific population. The lessons learned from this study will not only inform cancer control policy in NE Pennsylvania, but can inform other regional policymakers, who may benefit from using modelling for health economic evaluations before implementing national cancer control guidelines in their geographic area.

\section{Author affiliations}

${ }^{1}$ Institute for Technology Assessment, Massachusetts General Hospital, Boston, Massachusetts, USA

${ }^{2}$ Harvard Medical School, Boston, Massachusetts, USA

${ }^{3}$ Department of Biostatistics, Yale University School of Public Health, New Haven, Connecticut, USA

${ }^{4}$ Northeast Regional Cancer Institute, Scranton, Pennsylvania, USA

${ }^{5}$ Lombardi Comprehensive Cancer Center, Georgetown University, Washington DC, USA

Contributors PMM, SML and CYK had primary responsibility for the conception of the research. ACT, DFS, PMM, TRH, DTL and CYK contributed to the design of the research. KR and SML prepared the Northeast Pennsylvania data. ECD edited the manuscript. All authors contributed to manuscript preparation.

Funding This work was supported by the National Cancer Institute, National Institutes of Health grants to the CISNET Lung group (U01CA152956) and to CYK (K25CA133141) and funding from the Centers for Disease Control and Prevention.

Competing interests None declared.

Provenance and peer review Not commissioned; externally peer reviewed.

Data sharing statement No additional data are available.

Open Access This is an Open Access article distributed in accordance with the Creative Commons Attribution Non Commercial (CC BY-NC 4.0) license, which permits others to distribute, remix, adapt, build upon this work noncommercially, and license their derivative works on different terms, provided the original work is properly cited and the use is non-commercial. See: http:// creativecommons.org/licenses/by-nc/4.0/

\section{REFERENCES}

1. Siegel R, Ma J, Zou Z, et al. Cancer statistics, 2014. CA Cancer J Clin 2014;64:9-29. 
2. National Cancer Institute. National Lung Screening Trial Results 2010 (4 November 2010). http://www.cancer.gov/newscenter/ pressreleases/NLSTresultsRelease

3. Aberle DR, Adams AM, Berg CD, National Lung Screening Trial Research Team. Reduced lung-cancer mortality with low-dose computed tomographic screening. N Engl J Med 2011;365:395-409.

4. Moyer VA. Screening for lung cancer: U.S. Preventive Services Task Force recommendation statement. Ann Intern Med 2014;160:330-8.

5. Centers for Medicare \& Medicaid Services. Decision Memo for Screening for Lung Cancer with Low Dose Computed Tomography (LDCT) (CAG-00439N) 2015 (5 February 2015 and 29 July 2015). http://www.cms.gov/medicare-coverage-database/details/ nca-decision-memo.aspx?NCAld=274\&NcaName=Screening+for +Lung+Cancer+with+Low+Dose+Computed+Tomography+(LDCT) \&TimeFrame=7\&DocType=All\&bc=AQAAIAAAAgAAAA\%3d\%3d\&

6. Chahine T, Subramanian SV, Levy JI. Sociodemographic and geographic variability in smoking in the U.S.: a multilevel analysis of the 2006-2007 Current Population Survey, Tobacco Use Supplement. Soc Sci Med 2011;73:752-8.

7. Smieliauskas F, MacMahon $\mathrm{H}$, Salgia R, et al. Geographic variation in radiologist capacity and widespread implementation of lung cancer CT screening. J Med Screen 2014;21:207-15.

8. Jarvandi S, Yan Y, Schootman M. Income disparity and risk of death: the importance of health behaviors and other mediating factors. PLOS ONE 2012;7:e49929.

9. Koh HK, Nowinski JM, Piotrowski JJ. A 2020 vision for educating the next generation of public health leaders. Am J Prev Med 2011;40:199-202.

10. Garrett BE, Dube SR, Winder C, et al. Cigarette smoking-United States, 2006-2008 and 2009-2010. MMWR Surveill Summ 2013;62 (Suppl 3):81-4

11. Lesko SM. Cancer in Northeastern Pennsylvania: Incidence, Mortality and Survival for Common Cancers. http://www.cancernepa. org: Northeast Regional Cancer Institute, 2011.

12. Pennsylvania Department of Public Health. EpiQMS (19 August 2015). https://apps.health.pa.gov/EpiQMS/asp/SelectParams_ BRFSS Tbl_Region.asp

13. McMahon PM, Kong CY, Weinstein MC, et al. Adopting helical CT screening for lung cancer: potential health consequences during a 15-year period. Cancer 2008;113:3440-9.

14. McMahon PM, Kong CY, Johnson BE, et al. Estimating long-term effectiveness of lung cancer screening in the Mayo CT screening study. Radiology 2008;248:278-87.

15. McMahon PM, Kong CY, Johnson BE, et al. Chapter 9: the MGH-HMS lung cancer policy model: tobacco control versus screening. Risk Anal 2012;32(Suppl 1):S117-24.

16. Moolgavkar SH, Holford TR, Levy DT, et al. Impact of reduced tobacco smoking on lung cancer mortality in the United States during 1975-2000. J Natl Cancer Inst 2012;104:541-8.
17. McMahon PM, Kong CY, Bouzan C, et al. Cost-effectiveness of computed tomography screening for lung cancer in the United States. J Thorac Oncol 2011;6:1841-8.

18. Meza R, ten Haaf $\mathrm{K}$, Kong $\mathrm{CY}$, et al. Comparative analysis of 5 lung cancer natural history and screening models that reproduce outcomes of the NLST and PLCO trials. Cancer 2014;120:1713-24.

19. NRC (National Research Council). Health risks from exposure to low levels of ionizing radiation. BEIR VII phase 2. Washington DC: National Academy Press, 2005.

20. Egawa H, Furukawa K, Preston D, et al. Radiation and smoking effects on lung cancer incidence by histological types among atomic bomb survivors. Radiat Res 2012;178:191-201.

21. Centers for Disease Control and Prevention. Behavioral risk factor surveillance system survey data. Atlanta, GA: U.S. Department of Health and Human Services, Centers for Disease Control and Prevention, 2002-2006.

22. Ashmore JH, Lesko SM, Miller PE, et al. Association of dietary and supplemental iron and colorectal cancer in a population-based study. Eur J Cancer Prev 2013;22:506-11.

23. Centers for Disease Control and Prevention. Behavioral risk factor surveillance system survey data. Atlanta, GA: U.S. Department of Health and Human Services, Centers for Disease Control and Prevention, 2010.

24. Holford TR, Levy DT, McKay LA, et al. Patterns of birth cohort-specific smoking histories, 1965-2009. Am J Prev Med 2014;46:e31-7.

25. Levy DT, Graham AL, Mabry PL, et al. Modeling the impact of smoking-cessation treatment policies on quit rates. Am J Prev Med 2010;38(3 Suppl):S364-72.

26. Schauffler $\mathrm{HH}$, McMenamin $\mathrm{S}$, Olson $\mathrm{K}$, et al. Variations in treatment benefits influence smoking cessation: results of a randomised controlled trial. Tob Control 2001;10:175-80.

27. Levy DT, Friend K. A simulation model of policies directed at treating tobacco use and dependence. Med Decis Making 2002;22:6-17.

28. Clarke TC, Soler-Vila H, Fleming LE, et al. Trends in adherence to recommended cancer screening: the US population and working cancer survivors. Front Oncol 2012;2:190.

29. Wang Y, Midthun DE, Wampfler JA, et al. Trends in the proportion of patients with lung cancer meeting screening criteria. JAMA 2015;313:853-5.

30. Aberle DR, Adams AM, Berg CD, et al. Baseline characteristics of participants in the randomized national lung screening trial. $J$ Natl Cancer Inst 2010;102:1771-9.

31. Levy DT, Mabry PL, Graham AL, et al. Reaching Healthy People 2010 by 2013: a SimSmoke simulation. Am J Prev Med 2010;38(3 Suppl):S373-81.

32. Abrams DB, Graham AL, Levy DT, et al. Boosting population quits through evidence-based cessation treatment and policy. Am J Prev Med 2010;38(3 Suppl):S351-63. 